\title{
Reserpine for the treatment of refractory mania
}

\author{
I Eloff, MB ChB, DMH; W Esterhuysen, MB ChB, MMed (Psych) \\ Department of Psychiatry, Elizabeth Donkin Hospital, Walter Sisulu University, Port Elizabeth, South Africa
}

Corresponding author: I Eloff (ingrideloff@yahoo.com)

\begin{abstract}
We report a case of refractory mania treated successfully with reserpine. The patient was a 26-year-old man with bipolar I disorder, who had recurrent hospital admissions for manic episodes that failed to respond to multiple mood stabilisers and antipsychotics. The patient also suffered from hypertension. His manic symptoms improved markedly with the addition of reserpine to the treatment regimen. Reserpine is a centrally acting antihypertensive with anti-adrenergic properties. It has been found to reduce the duration of a manic episode rapidly in a small group of studies. This proved to be the case with this patient. Reserpine used in conjunction with other psychotropic medication may be considered in the treatment of refractory mania, but controlled trials are necessary to support our finding more generally.
\end{abstract}

S Afr J Psych 2014;20(1):31-32. DOI:10.7196/SAJP.456

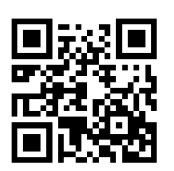

Reserpine, a Rauvolfia alkaloid with centrally acting anti-adrenergic properties, ${ }^{[1]}$ was previously widely used as a first-line drug in the treatment of mild to moderate hypertension, but has largely been replaced by other antihypertensives owing to its side-effect profile. We report a case of its use in the treatment of severe refractory mania.

\section{Case report}

$\mathrm{Mr}$ X, a 36-year-old, Xhosa-speaking male, presented to our unit for the first time in February 2003 at age 26 years. He believed that he was very wealthy and had grand plans of single-handedly building a sports stadium for the community. He was talkative with flight of ideas and underlying irritability. He fulfilled the Diagnostic and Statistical Manual of Mental Disorders (DSM)-IV criteria for a manic episode and scored 35 on the Young mania rating scale (YMRS). The patient was diagnosed with bipolar I disorder, manic phase, severe with psychotic features.

According to the patient and his family, this was his first presentation with mania and there was no history of previous depressive episodes, nor any family history of mental illness. Mr X's birth and developmental history was unremarkable and he matriculated successfully without ever having to repeat a year at school. He had been working as a machine operator for the past 5 years.

On admission there were no known medical conditions, nor any history of substance abuse. The patient stabilised within a few weeks of receiving a combination of haloperidol and sodium valproate. Upon his second admission in 2006, the patient again presented with manic symptoms. It was noted that his blood pressure (BP) was elevated, but he was not receiving treatment for hypertension. He was started on a single antihypertensive, together with a combination of haloperidol and sodium valproate, to which he responded. In 2009, the patient was admitted for the third time for a period of 5 months, during which he developed neuroleptic malignant syndrome while receiving a combination of sodium valproate and high doses of haloperidol. His manic symptoms did not respond to the single mood stabiliser; therefore, lithium carbonate was added to his treatment. The patient eventually stabilised on a combination of sodium valproate, lithium carbonate and clozapine. His hypertension was controlled on a combination of two antihypertensives. In October 2009, 3 months after discharge, $\mathrm{Mr} \mathrm{X}$ was readmitted for the fourth time with a manic episode and poorly controlled BP. The same combination of treatment that had proved to be effective during the previous admission was administered, but with poor response. A trial of aripiprazole and a subsequent trial of quetiapine also failed to stabilise his condition. He developed right-sided facial paralysis and was admitted to the medical department where a transient ischaemic attack was diagnosed. Further work-up revealed that the patient had left ventricular hypertrophy, but a relatively normal ejection fraction of $71 \%$.

In May 2010, the patient was treated with a course of 18 sessions of electroconvulsive therapy, with little effect. He was finally discharged, after 17 months in hospital, on a combination of carbamazepine, sodium valproate, lithium carbonate and olanzapine. His BP was controlled on a combination of four antihypertensives.

The patient was admitted for the fifth time in August 2011. He failed to respond to the treatment regimen that he had been taking previously when discharged. In November 2011, the patient was commenced on reserpine, considering its known ability to affect mood. Within the first two weeks, the patient's mood began to improve. He was quiet in the ward, slept at night and was much less intrusive. In March 2012, he was transferred to a long-term psychiatric hospital for ongoing supervision and management on a combination of reserpine (1 mg bd), sodium valproate $(1000 \mathrm{mg}$ bd) and olanzapine ( $20 \mathrm{mg}$ bd). He scored 8 on the YMRS upon discharge, and had no depressive features at the time.

\section{Discussion}

The use of reserpine as an antihypertensive has become unpopular, owing to its side-effect profile, most notably the development of depressive symptoms. However, as this case suggests, it is possible that this side-effect may have something to do with the positive response that we observed when using it for refractory mania. 


\section{CASE REPORT}

Reserpine, which has serotonin- and catecholamine-depleting properties, was shown to be effective in the treatment of mania in the early 1960s. ${ }^{[2]}$ It inhibits the uptake of monoamine neurotransmitters into storage granules, resulting in the metabolism of these neurotransmitters by monoamine oxidase. This depletion results in reserpine's antihypertensive effects and is likely to contribute to its association with depression. ${ }^{[3]}$

In 1986, Telner et al. ${ }^{[2]}$ described a preliminary investigation of 16 patients, diagnosed with bipolar I disorder and in a current manic phase, who were treated with a mood stabiliser for $1-8$ days with no resulting change in manic symptoms; $37.5 \%$ showed substantial improvement in symptoms with the addition of reserpine, while $43.8 \%$ showed moderate improvement within 48 hours of starting reserpine treatment. ${ }^{[2]}$ This suggested that, in conjunction with other psychotropic drugs, reserpine may have beneficial value in the rapid reduction of manic symptoms. A similar study in $1958^{[4]}$ showed that, in comparison with various other treatment methods, treatment with reserpine shortened the duration of manic episodes.

In our case, Mr X presented with a longstanding history of bipolar I disorder with refractory mania and several physical co-morbidities including hypertension. Upon his most recent admission, the patient had suffered refractory manic symptoms for approximately 18 months, despite treatment with several antipsychotic medications and mood stabilisers at adequate therapeutic doses. It is therefore unlikely that the resolution of symptoms resulted from the natural remitting course of a manic episode; it was only after the addition of reserpine that the manic symptoms improved rapidly. This supports the hypothesis that reserpine, used in conjunction with other psychotropic medication, may be beneficial in treating patients with refractory mania. However, controlled trials are required to support our finding more generally. It should also be determined whether there is an increased risk of a depressive episode associated with such treatment for refractory mania.

\section{References}

1. Hollister LE, Jones KP, Brownfield B. Chlorpromazine alone and with reserpine: Use in the treatment of mental diseases. Calif Med 1955;83(3):218-221.

2. Telner J, Lapierre Y, Horn E, Browne M. Rapid reduction of mania by means of reserpine therapy. Am J Psychiatry 1986;143(8):1058.

3. Huffman JC, Stern TA. Neuropsychiatric consequences of cardiovascular medications. Dialogues Clin Neurosci 2007;9(1):29-45.

4. Watt DC. The effect of reserpine on the duration of manic attacks. J Neurol Neurosurg Psychiatry 1958;21(4):297-300. 